\title{
Stretchable Electroadhesion for Soft Robots
}

\author{
Jürg Germann, Bryan Schubert and Dario Floreano
}

\begin{abstract}
With the ongoing rise of soft robots there emerges a need for new technologies that can cope with hyper-flexibility and stretchability. In this paper, we describe a new method for enabling controllable adhesion, namely electroadhesion, for use in soft robots. We present a method to manufacture stretchable electroadhesive pads and characterize their performance when stretching the pad more than double its original length. Our results suggest that the normal detachment force per area slightly decreases with the stretching, while the shear detachment force per area increases with the stretch ratio. These results imply that stretchable electroadhesive pads have higher adaptivity than non-stretchable pads because their mechanical stiffness and adhesive forces can be controlled through stretching.
\end{abstract}

\section{INTRODUCTION}

Recently, there has been great interest in the development of novel bio-inspired soft robots [1]. Soft robots have many potential applications because they can adapt to unpredictable environments and operate safely with humans [2]. From a technical point of view, soft robots are expected to solve a variety of tasks in a mechanically simpler and computationally more efficient way compared to traditional hard robots [3]. By definition, soft robots should be hyper flexible and highly adaptive, and their functionality should be enabled and not hindered by large deformations of body parts [4].

In this paper we focus on creating controllable adhesion for soft robots by extending the electroadhesive principle to highly deformable materials. Controllable adhesion is a desirable functionality for many robots, including wall-climbing robots, grasping robots, or more general robots that manipulate objects or surfaces. Research on electroadhesion has shown the many advantages of this technology: it attaches to a wide range of substrates and surfaces, generates high clamping forces, is electrically controllable and mechanically simple, lightweight and compliant; also, power consumption is low and operation is quiet [5].

Electroadhesion has been used in industry for several years in applications such as grippers [6], [7], clothing manufacturing [8], electrostatic chucks [9], [10], and in the last several years as an attachment method for climbing robots [5] and modular robots [11], [12]. Also, patents are held on the technology [15], [16], [17] and its application in several areas, including medical devices [18], climbing robots [19], grippers [20], or extension ladder stability devices [21].

However, to the best of our knowledge there has not been any discussion of how to fabricate stretchable electroadhe-

J. Germann, B. Schubert and D. Floreano are with the Laboratory of Intelligent Systems, School of Engineering, École Polytechnique Fédérale de Lausanne (EPFL), 1015 Lausanne, Switzerland

Email: jurg.germann@epfl.ch

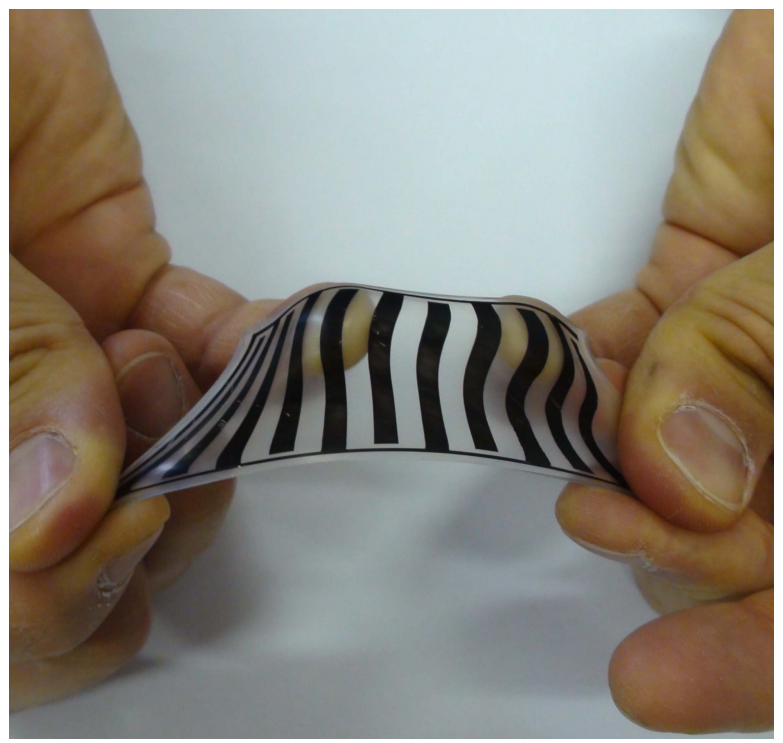

Fig. 1. Fabricated stretchable electroadhesive pad being deformed.

sive pads, nor has there been any characterization of how stretchable pads perform. In order to extend the usability of electroadhesion to soft (and especially highly elastic) robots, we present a method to manufacture stretchable electroadhesive pads such as the one shown in Fig. 1, and characterize their performance under various stretching conditions.

In the following sections we first explain the basic theory of electroadhesion, and then describe in detail our design and fabrication method. After introducing the experimental setup used to characterize both normal and shear detachment, we present and discuss the obtained results.

\section{THEORETICAL BACKGROUND}

Electroadhesive devices are composed of electrodes with alternating charges, as shown in Fig. 2. The electric fields that arise between the alternating electrodes attract free charges in conductors and cause polarization in insulating substrates that are brought near the electroadhesive pads. The induced and polarized charges are responsible for the electrostatic forces that result in adhesion. We will focus mainly on the insulating case because it is the most prevalent in natural environments.

When the contacting substrate is an insulator, determining the force of attraction is non-trivial because calculating the electrostatic forces requires knowing the exact profile of the electric field throughout the system, as shown in Fig. 2a [13]. However, even without a complete solution, we can still 
(a)

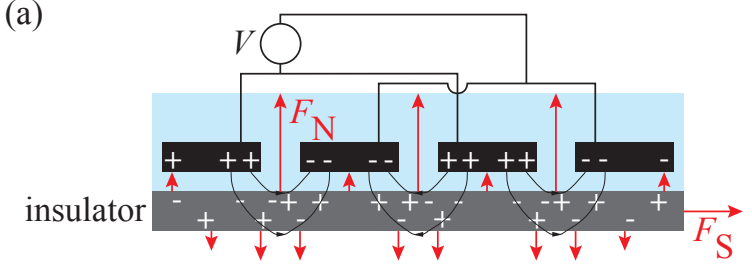

(c)

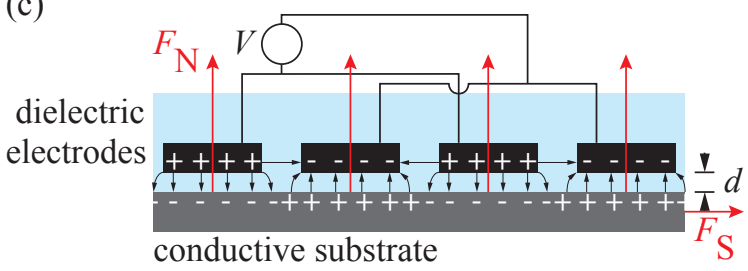

(b)

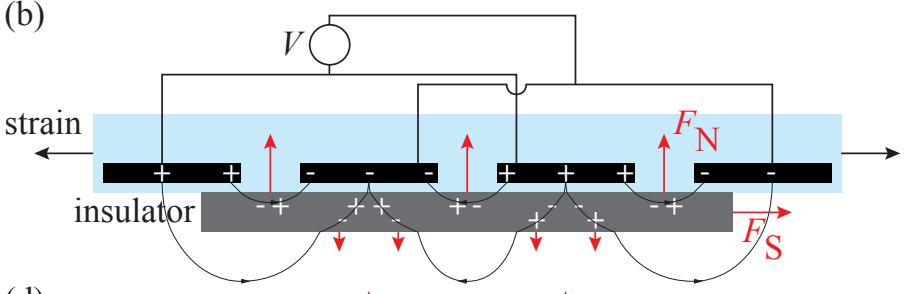

(d)

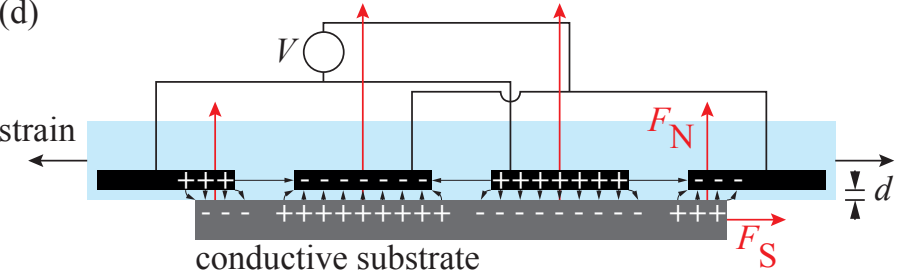

Fig. 2. Operating principle of stretchable electroadhesive pads. Pads are composed of a stretchable dielectric that encapsulates stretchable electrodes. When a DC voltage is applied to the electrodes, the generated electric field causes (a) polarization of nearby insulators or (c) surface charging of nearby conductive substrates. This generates an attractive force, $F_{\mathrm{N}}$, between the substrate and pad, and allows the pads to withstand applied shear forces, $F_{\mathrm{S}}$. Straining of the stretchable pads changes their geometry, (b) decreasing the normal force between the pad and insulating substrates, and (c) increasing the normal force when attached to conductive substrates.

make some general statements about how electroadhesion behaves with insulating materials.

Polarization leads to weaker attractive forces than pure charge separation because of repulsion from like charges in the induced dipoles. The strongest forces are generated within the boundaries between the oppositely charged pads, as shown in Fig. 2a. Within the boundaries, the electric field strength is highest, and some field lines do not fully penetrate the thickness of the dielectric. This second point means that some field lines do not generate opposing forces on the opposite side of the insulator, so the net attractive force is higher [22]. Therefore, if we want to strongly attach to insulating substrates, we should create electroadhesive pads with more boundary regions. However, there is a limit to the number of boundaries that can be used before the decrease in electrode area has a negative effect on the electroadhesion force [22].

For stretchable electroadhesive pads, the electrostatic forces will vary depending on how the pad is deformed. To examine the effects of strain on electroadhesion, we assume that the pad material is incompressible, meaning that the volume of the pads is conserved. This leads to the following expression,

$$
\left(s_{\mathrm{x}}+1\right)\left(s_{\mathrm{y}}+1\right)\left(s_{\mathrm{z}}+1\right)=\lambda_{\mathrm{x}} \lambda_{\mathrm{y}} \lambda_{\mathrm{z}}=1,
$$

where $s$ values are the strains and $\lambda=s+1$ values are the stretch ratios in the $x$-, $y$ - and $z$-directions.

For adhesion to an insulating substrate, stretching will have a negative impact on the electroadhesion mechanism. As the pads are stretched, the gap between electrodes increases linearly, decreasing the electric field strength, and therefore the magnitude of polarization. Furthermore, the number of boundary regions in contact with a given substrate area may decrease if they are stretched past the edge of the substrate, see Fig. 2.

It is also interesting to consider adhesion to conductive substrates because there are simple expressions that allow us to clearly see the influence of stretching. The theory for conductive substrates is based on the assumption that charge separation is uniform within the area across from the pads (see Fig. 2c). The electroadhesive force in the direction normal to the pad, $F_{\mathrm{N}}$, is then,

$$
F_{\mathrm{N}}=\frac{1}{2} A \varepsilon_{0} \varepsilon_{\mathrm{r}}\left(\frac{V}{2 d}\right)^{2}
$$

where $A$ is the pad area, $V$ is the applied voltage, $d$ is the dielectric thickness, $\varepsilon_{0}$ is the permittivity of free space and $\varepsilon$ is the permittivity of the dielectric layer [12]. To see how stretching affects this force, we combine Eqn. 1 and Eqn. 2,

$$
F_{\mathrm{N}}=\frac{1}{2} A_{0} \varepsilon_{0} \varepsilon_{\mathrm{r}}\left(\frac{V}{2 d_{0}}\right)^{2} \lambda_{\mathrm{x}}^{3} \lambda_{\mathrm{y}}^{3}=F_{\mathrm{N} 0} \lambda_{\mathrm{x}}^{3} \lambda_{\mathrm{y}}^{3},
$$

where $A_{0}, d_{0}$ and $F_{\mathrm{N} 0}$ are respectively the area, dielectric thickness and electroadhesive force of the unstretched pads. As this equation shows, the overall force of a pad increases greatly as the pad is stretched. The increase of the total force comes from an increase in the pad area and a decrease in the dielectric thickness. However, the force per area, or pressure, increases only as a result of the change in dielectric thickness,

$$
P_{\mathrm{N}}=\frac{1}{2} \varepsilon_{0} \varepsilon_{\mathrm{r}}\left(\frac{V}{2 d_{0}}\right)^{2} \lambda_{\mathrm{x}}^{2} \lambda_{\mathrm{y}}^{2}
$$

Eqns. 3 and 4 are presented for completeness, but they are not experimentally validated in this paper. This is namely because they are extensions of Eqn. 2, which is commonly used in electroadhesion literature.

The discussion so far has only mentioned the normal force that can be expected from electroadhesion. However, for most applications we are also interested in the shear force. Generally the shear force is regarded as a function of the normal force,

$$
F_{\mathrm{S}}=\mu_{\mathrm{f}} F_{\mathrm{N}}
$$


where $\mu_{\mathrm{f}}$ is the coefficient of friction between the pad and the substrate [5]. For rigid pads, it may be acceptable to assume a constant coefficient of friction for a given substrate. However, with stretchable pads, this assumption is likely no longer valid because stretching will change the surface properties of the pad material, such as its stiffness and roughness, which will impact it's ability to make contact with a given substrate.

\section{DESIGN AND FABRICATION}

The electroadhesive pads, see Fig. 3, use a standard interdigitated design, or ladder, with equal track width, $w$, and spacing, $g$, of 400, 600 and $800 \mu \mathrm{m}$ fit into a $2 \times 2 \mathrm{~cm}$ area. The ladder design is chosen because it is known to adhere well to insulating substrates [22]. This is because it has many boundaries that enhance polarization, as discussed in the theory section. For adhering to conductive substrates, a simpler design with only two pads that maximizes the pad area would be more appropriate.

We chose multiple track widths and spacings in order to evaluate the effect of the number of boundaries on the electroadhesive strength, where the smaller tracks have more boundary zones. The average thickness of the tracks, $t$, and dielectric layer, $d$, are kept constant across samples and they are $t=22 \pm 2 \mu \mathrm{m}$ and $d=19 \pm 6 \mu \mathrm{m}$, respectively, as measured by optical microscopy (Olympus IX73) (see Fig. 3f).

When designing electroadhesive pads, the resistance of the tracks is important because it impacts the charging time required to reach full strength. For rigid pads, good conductors, such as metal films, can be used to minimize the resistance. However, if the pads need to stretch, we must use a conductive material that can undergo large strains with minimal increase in resistivity. Our solution is to use a mixture of rubber (Ecoflex 00-30 from Smooth-On, Inc.) and carbon black (CB) (Vulcan XC72 from Cabot Corporation, 20\% by weight). This combination has relatively high resistivity of $0.381 \Omega-\mathrm{m}$ at $0 \%$ strain, but it has a low increase in resistivity of only $10 \%$ at strain as high as $130 \%$.

For the fabrication, we used a sequential casting method similar to [14] (see Fig. 3a-d) that can be used with a variety of materials and pad designs. First, a conductive rubber is scraped over an etched stainless steel mold using a plastic blade. This leaves the conductive mixture only in the wells. Then, a pure rubber (Ecoflex 00-30) layer of approximately $2 \mathrm{~mm}$ thickness is poured over the mold and left to cure for 4 hours at room temperature. After curing, the pure rubber and conductive rubber tracks are well bonded and can be peeled from the mold. Finally, to encapsulate the tracks, a thin layer of rubber (Ecoflex 00-30) is spin-coated over the entire sheet and left to cure for 4 hours at room temperature.

\section{EXPERIMENTAL SETUP}

In order to test our developed samples under different strains, we fabricated a stretching device (stretcher). The

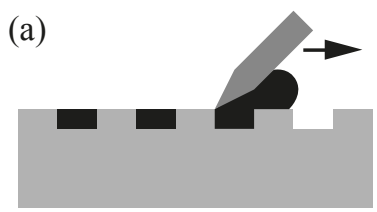

(b)

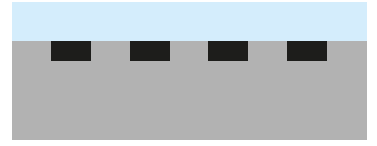

(e)

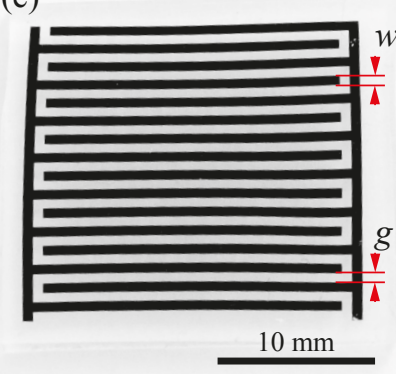

(c)

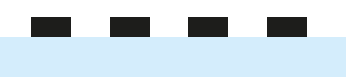

(d)

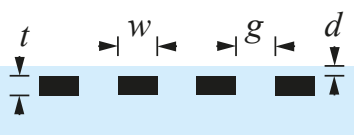

(f)

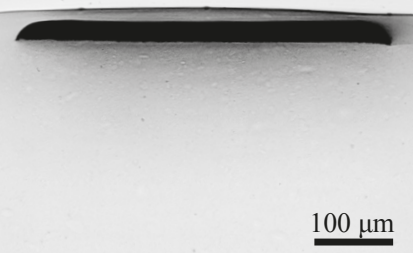

Fig. 3. Fabrication consists of (a) scraping conductive Ecoflex/CB mixture into a stainless steel mold to form the conductive traces, (b) coating the traces with pure Ecoflex, (c) de-molding the Ecoflex substrate with the bonded traces and (d) spin-coating the conductive traces with an encapsulating layer of pure Ecoflex. (e) Completed stretchable electroadhesive pad. (f) Cross-section showing encapsulated conductive trace.

custom built stretcher can obtain stretch ratios ranging from 1 to 5 independently in both directions. The pads are attached to the stretcher by acrylic clamps that squeeze the edge of the film. Figure $4 \mathrm{a}$ shows the mounted pad on a stretcher in unstretched and stretched state.

For the adhesion experiments we mount the stretcher to a perforated acrylic plate in order to maintain fixed stretch ratios. We then test the normal (pull-off) and shear detachment forces of the electroadhesive pads from an insulating substrate (cellulose). For the normal force tests, we use the setup shown in Figure 4b. A probe consisting of a $20 \times 20 \mathrm{~mm}^{2}$ cellulose surface is mounted on a force sensor (Nano 17, 6-axis force/torque sensor from ATI Industrial Automation) which in turn is connected to a linear stage (T-LSR150B from Zaber Technologies). We use this setup to control position and displacement of the probe while recording the adhesion force in the z-direction. A single measurement is performed as follows: the probe approaches the electroadhesive pad until a preload force of approximately $0.15 \mathrm{~N}$ is reached. After $1 \mathrm{~s}$ it is retracted at a speed of $500 \mu \mathrm{m} \mathrm{s}^{-1}$ until the probe detaches from the pad.

For the shear tests, we rearrange the setup as shown in Figure 4c. The probe is connected via string to the force sensor. In these experiments, we record the force in the $x$-direction. A single measurement is run as follows: the probe is placed on an electroadhesive pad manually. No other preload force than the weight of the probe $(3.3 \mathrm{~g})$ is added. Subsequently, the probe is pulled using the linear stage at a 
(a)

(b)

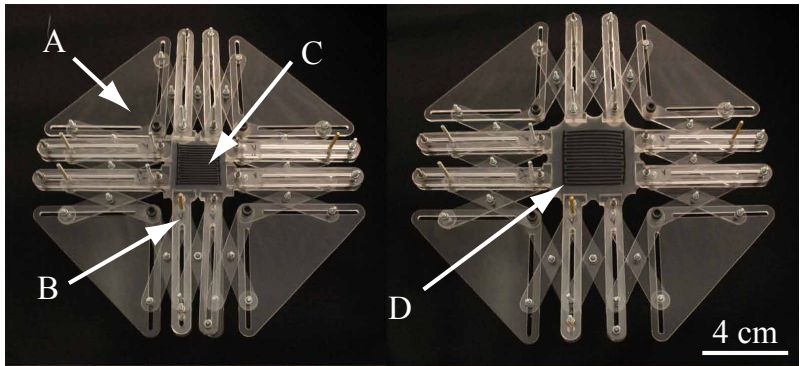

(c)
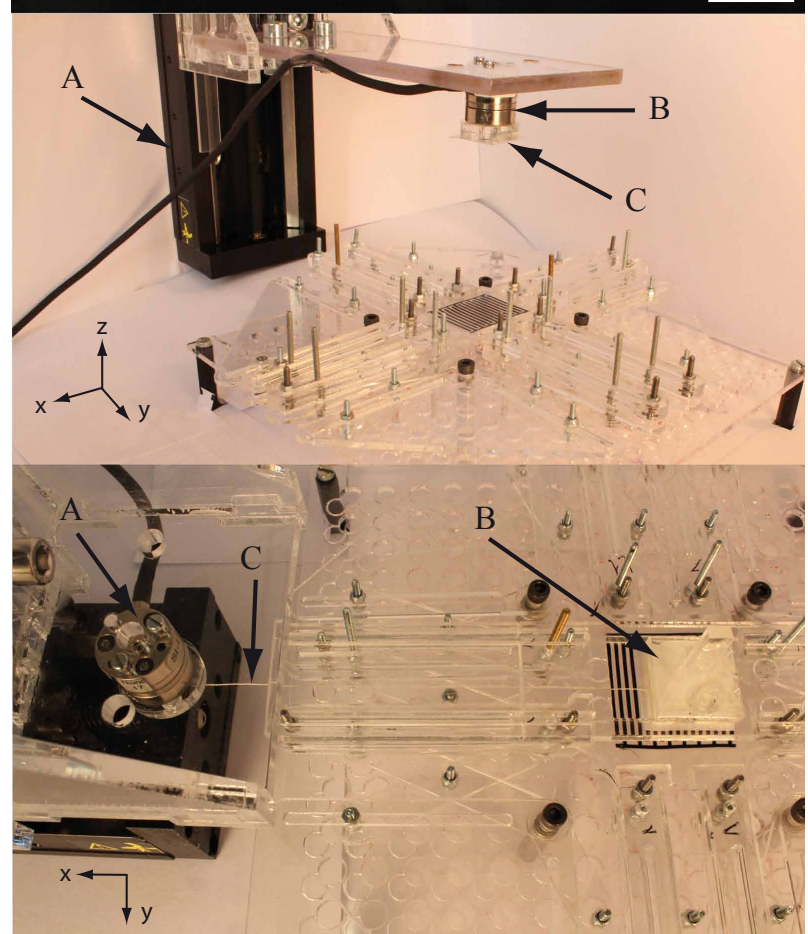

Fig. 4. Experimental setup: (a) A: stretcher, B: connecting piece to grab electroadhesive pad, C: electroadhesive pad unstretched, D: electroadhesive pad stretched; (b) Setup to measure normal force: A: linear stage, B: force sensor, C: probe; (c) Setup to measure shear force: A: force sensor, B: probe, $\mathrm{C}$ : string to connect probe with force sensor (and linear stage).

speed of $500 \mu \mathrm{m} \mathrm{s}^{-1}$ until the probe detaches from the pad. The probe is constrained by an acrylic frame that prevents it from rotating during pulling.

Both normal and shear force measurements are performed for five different strain settings in the $x$-direction $(25 \%$, $50 \%, 75 \%, 100 \%$ and $125 \%)$. In all of these experiments, the probe area is smaller than the total pad area, so the force measured only represents a portion of the total pad force. The strain in the $y$-direction is kept constant at $50 \%$. In total we test 9 different samples with 3 different electrode-gap-widths $(0.4,0.6$ and $0.8 \mathrm{~mm})$. The operating voltage for the experiments is set to $0 \mathrm{~V}$ or $2000 \mathrm{~V}$. For each different setting (sample, strain, voltage), we run 5 experiments consecutively.

\section{RESULTS \& DISCUSSION}

In this section, we characterize the performance of stretchable electroadhesive pads in terms of their normal and shear force under different strains.

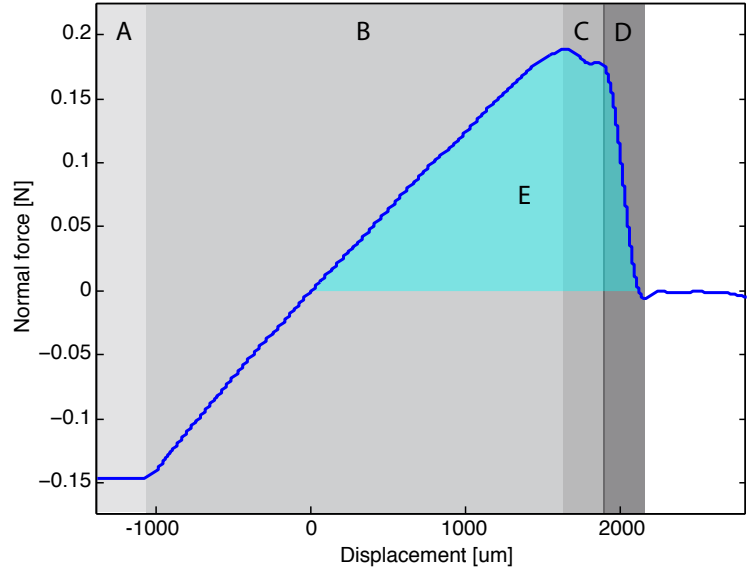

Fig. 5. Example force-displacement profile of a normal force measurement (voltage supply 2000V): (A) preloading phase, (B) pulling phase, (C) peeling phase, (D) detachment, (E) area under curve corresponding to required work for detachment.

\section{A. Normal force results}

An example of the typical normal force-displacementprofile is shown in Fig. 5. As the figure indicates, there are four different phases of a measurement: preloading, pulling, peeling and detachment. Note that the peeling phase is very short and always leads to complete detachment. In order to compare the different samples and strain ratios, we extract the maximum detachment force as well as the required work to detach from the force-displacement plots (see Fig. 5).

Fig. 6 plots the normal detachment force and work, respectively, under different conditions. The magnitude of the detachment force (and also work) is relatively low. With increasing strain the force as well as the work decreases further. This behavior is due to both electrical and mechanical effects. First, because the electrode-gap-width increases when stretching the pad, the electric field in the gap and thus the electrostatic holding force decreases. Second, the stretching leads to a lower total number of boundaries that are within the contact area of the probe, which again decreases the total electroadhesion strength. Third, increasing the strain effectively stiffens the pads, which impacts the pad's ability to conform to the attaching surface. This results in an increased distance between the electrodes and substrate, and thus in lower electrostatic forces. However, this also impacts the contact area of the pad, and therefore the natural adhesion of the rubber, as supported by the zero voltage data. Finally, the imposed strain decreases the thickness of the electroadhesive pad, this decreases the peeling force necessary for detachment [23]. This statement is further supported by the decrease of the required work to detach with higher strains.

Varying the size of the electrode-gap-width only has a small effect on the observed force and work. The theory predicts that as the electrode-gap-width increases, the electroadhesion force should decrease. However, we only clearly observe this in the measurements taken with the highest strain. It is difficult to observe this trend at smaller strains 
(a)

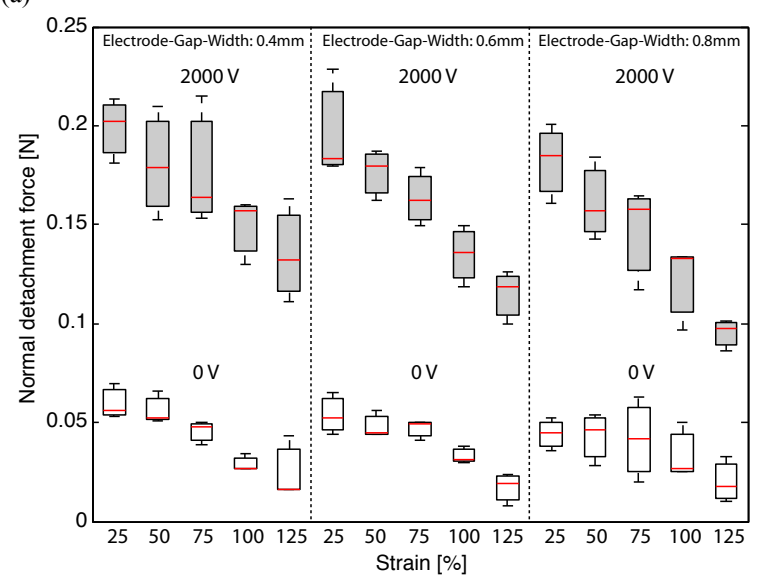

(b)

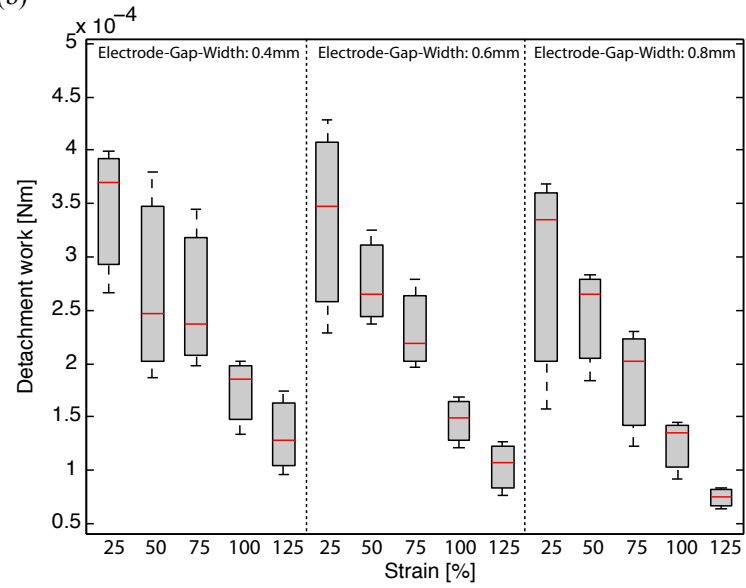

Fig. 6. (a) Normal detachment force versus strain and different electrodegap-width with voltage supply of $2000 \mathrm{~V}$ and $0 \mathrm{~V}$, (b) normal detachment work versus strain and different electrode-gap-width (voltage supply 2000V).

because the inherent tackiness of the rubber dominates the measured forces.

\section{B. Shear force results}

An example of a typical shear-force-displacement-profile is shown in Fig. 7. The different phases of a measurement include: shearing, stick-slip and detachment. During the shearing phase the probe is pulled by the linear stage, forcing the pad to stretch. In the stick-slip phase the electroadhesive pad starts to buckle while the probe detaches for short amounts of time before completely detaching. We extract from the data the maximum force (detachment force), as well as the required work to detach for the various samples and strains.

The resulting shear detachment force and work under different conditions is shown in Fig. 8. Unlike the normal measurements, the shear force and work increase with increased pre-strain. This trend is counter to Eqn. 5, which says that the shear force should be proportional to the normal force. However, this equation does not take into account the behavior of the freely supported elastic pad area that surrounds the shear probe. During testing, we observed that

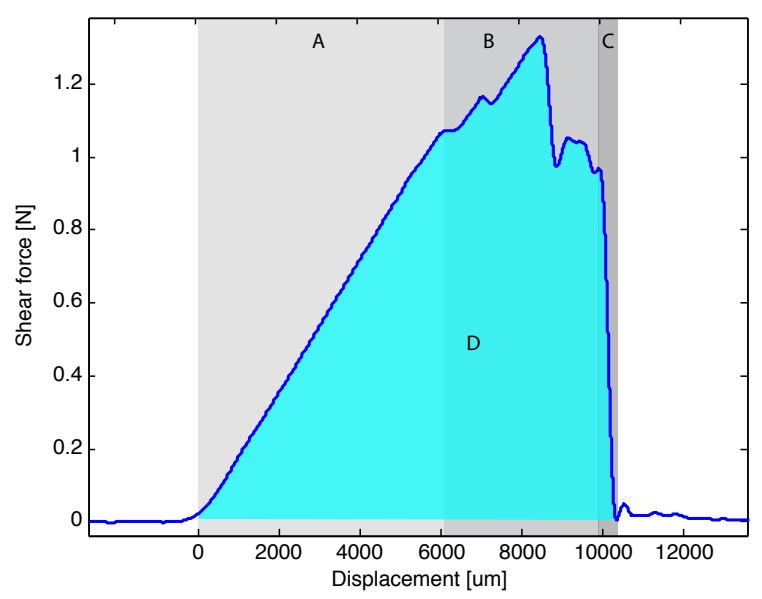

Fig. 7. Example force-displacement profile of a shear force measurement (voltage supply 2000V): (A) shearing phase, (B) stick-slip phase, (C) detachment phase, (D) area under curve corresponding to required work for detachment.

the detachment occurred when the pad started to buckle. Therefore, the increase in shear force with strain is likely due to higher required buckling forces as the pad is stretched. This is further supported by the zero-voltage measurements that show a similar trend.

\section{CONCLUSIONS}

In this paper, we demonstrated the feasibility and successful functioning of stretchable electroadhesive pads. We introduced a simple fabrication method to obtain pads that can be highly stretched (more than double their original size), and we characterized the pads under different strains. Our results show that normal force per area decreases with increasing strain, both due to electrical and mechanical effects. On the other hand, the shear force per area increases with strain, which can be explained because of increased buckling resistance of the pads during stretching. While we acknowledge that the observed trends are highly influenced by mechanical effects, the electroadhesion provides an enhancement of the magnitude of the observed forces by helping the soft rubber pads make more intimate contact with the rough cellulose substrates.

It can be concluded from the results that the stretchable electroadhesive pads have higher adaptivity to a given task compared to non-stretchable pads. This is because the stretchable pads are adaptable in terms of their mechanical stiffness as well as their adhesive force. Not only are the normal and shear force per area tunable with stretching, the total holding force of a pad is also tunable because the pad surface area is variable.

The advantages afforded by stretchable pads may improve the function of electroadhesive devices in existing applications, such as medical devices [18], grippers [20] or climbing robots [19]. Stretchable pads will also enable the inclusion of electroadhesion in new areas, such as soft robotics, where traditional rigid pads are fundamentally unsuitable. Furthermore, for soft robots that are expected 
(a)

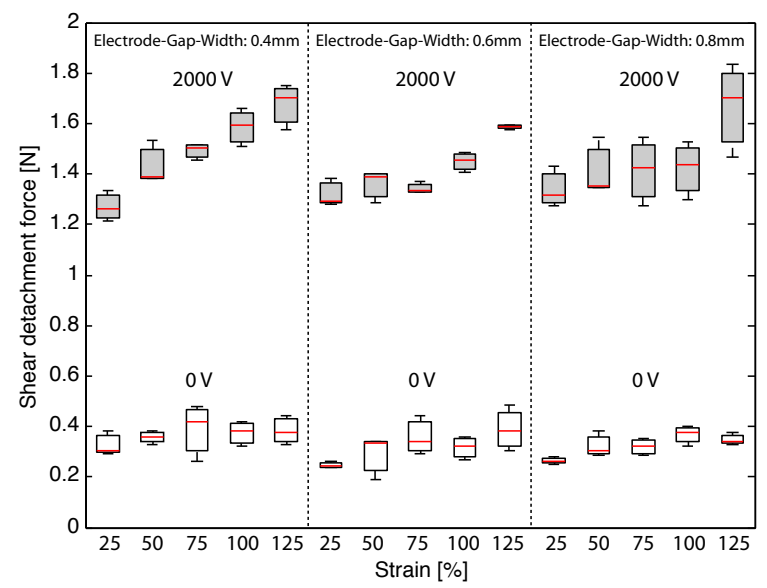

(b)

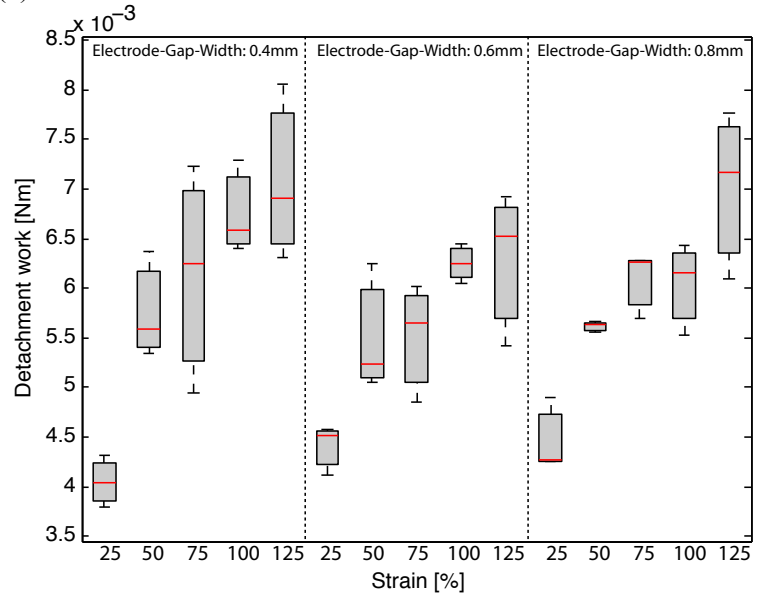

Fig. 8. (a) Shear detachment force versus strain and different electrodegap-width with voltage supply of $2000 \mathrm{~V}$ and $0 \mathrm{~V}$, (b) shear detachment work versus strain and different electrode-gap-width (voltage supply 2000V).

to operate in close proximity with humans, electroadhesion may be an especially well-suited adhesion technology since it attaches to a wide range of materials, including organic materials. Of course, proper insulation dimensions and operating voltages will need to be considered in order to make safe devices.

\section{ACKNOWLEDGMENTS}

This research was supported by the Swiss National Science Foundation through the National Center of Competence in Research Robotics. The authors would like to thank Constantinos Stergiopulos for his contribution on the hardware developments of this work. Furthermore, the authors gratefully acknowledge Andrea Maesani and Jun Shintake for their helpful comments on this manuscript.

\section{REFERENCES}

[1] R. Pfeifer, H. Marques, F. Iida, "Soft Robotics: The Next Generation of Intelligent Machines", Proceedings of the Twenty-Third International Joint Conference on Artificial Intelligence, pp. 5-11, 2013.
[2] D. Trivedi, C.D. Rahn, W.M. Kier and I.D. Walker, "Soft robotics: biological inspiration, state of the art, and future research", Applied Bionics and Biomechanics, vol. 5, no. 3, pp. 99-117, 2008.

[3] S. Kim, C. Laschi, and B. Trimmer, "Soft robotics: a bioinspired evolution in robotics", Trends in Biotechnology, vol. 31, no. 5, pp. 287-294, 2013

[4] H. Lipson, "Challenges and Opportunities for Design, Simulation, and Fabrication of Soft Robots", Soft Robotics, vol. 1, no. 1, pp. 21-27, 2013.

[5] H. Prahlad, R. Pelrine, S. Stanford, J. Marlow and R. Kornbluh, "Electroadhesive robots-wall climbing robots enabled by a novel, robust, and electrically controllable adhesion technology," IEEE International Conference on Robotics and Automation, pp. 3028-3033, 2008.

[6] G. Monkman, "Electroadhesive microgrippers" Industrial Robot: An International Journal, vol. 30, no. 4, pp. 326-330, 2003.

[7] G. J. Monkman, "Robot Grippers for Use With Fibrous Materials", The International Journal of Robotics Research, vol. 14, no. 2, pp. 144-151, 1995.

[8] G. J. Monkman, P. M. Taylor, and G. J. Farnworth, "Principles of electroadhesion in clothing robotics", International Journal of Clothing Science and Technology, vol. 1, no. 3, pp. 14-20, 1989.

[9] G. I. Shim and H. Sugai, "Dechuck Operation of Coulomb Type and Johnsen-Rahbek Type of Electrostatic Chuck Used in Plasma Processing" Plasma and Fusion Research, vol. 3, pp. 051-051, 2008.

[10] K. Asano, F. Hatakeyama, and K. Yatsuzuka, "Fundamental Study of an Electrostatic Chuck for Silicon Wafer Handling", IEEE Transactions on Industry Applications, vol. 38, no. 3, pp. 840-845, 2002.

[11] M. E. Karagozler, J. D. Campbell, G. K. Fedder, S. C. Goldstein, M P. Weller, and B. W. Yoon, "Electrostatic latching for inter-module adhesion, power transfer, and communication in modular robots", IEEE International Conference on Intelligent Robots and Systems, pp. 2779-2786, 2007.

[12] J. Germann, M. Dommer, R. Pericet-Camara and D. Floreano, "Active connection mechanism for soft modular robots," Advanced Robotics, vol. 26, pp. 785-798, 2012.

[13] H. Shen, R. Liu, R. Chen and J. He, "Modeling of attraction force generated by interdigitated electrodes for electroadhesive robots,' IEEE International Conference on Industrial Informatics, pp. 678-681, 2012.

[14] C.-Y. Liu and J.-W. Choi, "Precision patterning of conductive polymer nanocomposite using a laser-ablated thin film," Journal of Micromechanics and Microengineering, vol. 22, pp. 045014, 2012.

[15] R.E. Pelrine, H. Prahlad, J.S. Eckerle, R.D. Kornbluh, and S. Stanford, "Electroadhesion", US Patent 7773363 B2, 2010

[16] R.E. Pelrine, H. Prahlad, J.S. Eckerle, R.D. Kornbluh, and S. Stanford, "Electroadhesive devices", US Patent 8125758 B2, 2012

[17] H. Prahlad, R.E. Pelrine, A. Wong-Foy, R.D. Kornbluh, and B.K McCoy, "Materials for electroadhesion and electrolaminates", US Patent 0010398 A1, 2013

[18] S. MacLaughlin, "Electroadhesive Medical Devices", US Patent 0249 553 A1, 2010

[19] R.E. Pelrine, H. Prahlad, R.D. Kornbluh, P. D. Lincoln, and S. Stanford, "Wall Crawling Robots", US Patent 0059298 A1, 2010

[20] H. Prahlad, R.E. Pelrine, P.E. Garcia, R. Mahoney and J. Eckerle, "Electroadhesive gripping", US Patent 0193362 A1, 2011

[21] H. Prahlad, R.E. Pelrine, P. Simeonov, and H. Hsiao, "Electroadhesion device for improving extension ladder stability", US Patent 0315477 A1, 2011

[22] J. U. Jeon and T. Higuchi, "Electrostatic suspension of dielectrics," IEEE Transactions on Industrial Electronics, vol. 45, no. 6, pp. 938946, 1998.

[23] K. Kendall, "Thin-film peeling-the elastic term," Journal of Physics D: Applied Physics, vol. 8, pp. 1449-1452, 1975. 